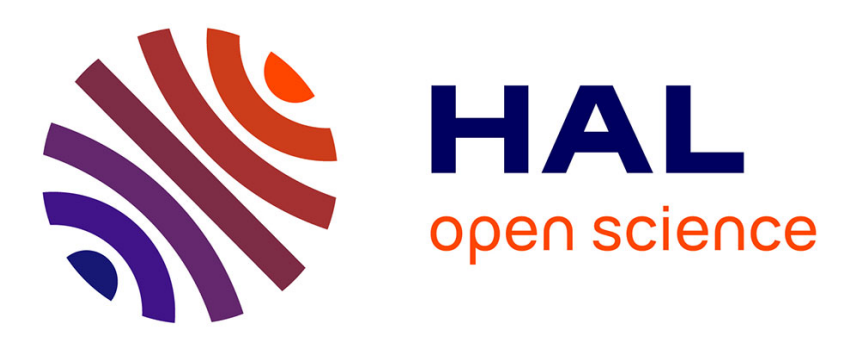

\title{
A non-equilibrium approach to processing Hopkinson Bar bending test data: Application to quasi-brittle materials
}

\author{
Franck Delvare, Jean-Luc Hanus, Patrice Bailly
}

\section{- To cite this version:}

Franck Delvare, Jean-Luc Hanus, Patrice Bailly. A non-equilibrium approach to processing Hopkinson Bar bending test data: Application to quasi-brittle materials. International Journal of Impact Engineering, 2010, 37 (12), pp.1170-1179. 10.1016/j.ijimpeng.2010.07.001 . hal-00601549

\section{HAL Id: hal-00601549 \\ https://hal.science/hal-00601549}

Submitted on 20 Jun 2011

HAL is a multi-disciplinary open access archive for the deposit and dissemination of scientific research documents, whether they are published or not. The documents may come from teaching and research institutions in France or abroad, or from public or private research centers.
L'archive ouverte pluridisciplinaire HAL, est destinée au dépôt et à la diffusion de documents scientifiques de niveau recherche, publiés ou non, émanant des établissements d'enseignement et de recherche français ou étrangers, des laboratoires publics ou privés. 


\title{
A non equilibrium approach to processing Hopkinson Bar bending test data: application to quasi-brittle materials
}

\author{
F. Delvare, J.L. Hanus, P. Bailly \\ Institut PRISME, ENSI de Bourges 88 Boulevard Lahitolle, 18020 BOURGES Cedex, FRANCE
}

\begin{abstract}
The aim of this paper was to describe a method of analysing the test data recorded during a Hopkinson Bar bending test. This three-point dynamic bending test was designed for testing the strength of materials under dynamic loads. It is carried out on a specimen consisting of a beam placed on two supports, which is subjected to an impact. The use of Hopkinson Bar as supports makes it possible to determine the forces and displacements at these points. An analytical solution for the transient response of a long beam subjected to a transverse impact was used to determine the impact force and the displacement. This procedure applies for the first few instants when the motions generated by the impact have not yet reached the supports, and the mechanical state of the specimen is identical to that of an unsupported beam. It is suitable for use with quasi-brittle materials for which failure occurs at the very beginning of the test. The material strength is determined at the time of failure, which is characterised by a sudden decrease in the bending moment. The results of a test in which a quasi-brittle material was loaded up to failure are presented and analysed as outlined above. The results obtained confirm the relevance of the present method.
\end{abstract}

Key words: Transient response, non equilibrium state, bending test, Hopkinson Bar, quasi-brittle material 


\section{Introduction}

Obtaining information about the dynamic behaviour of building materials such as concrete and some geomaterials is of critical importance to be able to predict how buildings will resist accidental events such as shocks. There exist several experimental methods for investigating the mechanical behaviour of quasi-brittle materials subjected to dynamic loads [1]. Fracture tests have been performed on quasi-brittle slabs, where the main load involved was a bending load, using an impact tube or blast wave methods [2] [3]. Other methods involve the use of Split Hopkinson (Kolsky) Pressure Bar (SHPB) [4], [5]. Although recent advances have improved the performances of test apparatuses and the accuracy of the measurements, the SHPB method is still particularly appropriate in this context because it can be used to apply a dynamic load to a specimen and to measure both the impact force and the impact velocity at the impact point. However, special precautions are required when dealing with brittle materials because failure of these materials occurs at very low strains. It is necessary in particular to be able to perform highly precise time shifting procedures to the signals measured [6]. The tensile strength of quasi-brittle materials differs considerably from their compression strength. Several modified set-ups have been presented for performing dynamic tensile tests [7], spalling tests [8] and Brazilian tests [9]. Compression tests have also been performed under confined conditions using a pressurized cell [10] or a metal ring [11].

The dynamic three-point bending test is widely used to determine the tensile strength of materials such as quasibrittle materials, because it is often easier to apply than direct tensile tests. The idea of performing three-point bending tests with Hopkinson bar has been mainly applied so far to determine the toughness of notched specimens [12], [13], [14], [15]. Hopkinson bar tests yield more information than Charpy tests, and the interpretation of the measurements is simplified [15] [16]. The three-point bending test can be used to closely analyse experimental measurements in order to obtain as much information as possible about the various phases of the response, including the rupture phase in particular.

In a general manner, the procedure for processing the dynamic response of a specimen loaded at its midpoint depends on the relationship between the duration of the test, defined as time up to complete failure, and the characteristic times of the structure. These responses are usefully divided into three main types, depending on the test duration.

- Quasi-static response : duration long in comparison with the structural characteristic times.

The reaction of the supports is equal to the half of the impact force. In this case the stresses and strains can be determined using a classical method. The fracture stress can then be obtained from the force or displacement measurements. Most toughness tests designed to determine the stress intensity factor are interpreted under the assumption that the quasi-static state of equilibrium is valid, taking the analytically determined stress field in a notched specimen [15], [17].

- Dynamic response : duration is short or comparable to the time needed for a wave to propagate to the specimen ends and return back to the impact point. This makes it necessary to take the transient motion of the beam into account.

- If the reaction of the supports is neither zero nor equal to the half of the impact force, we will call this dynamic response a quasi-impulsive response since duration is comparable to the time needed for a wave to propagate to the specimen ends and return back to the impact point. The transient response of the test specimen can be expressed using a model with a single degree of freedom (SDOF) [18], [19]. To account for the inertia of the test specimen, the Rayleigh approximation for the eigenfrequency can be used. Since the deformed structure is assumed to be the same as that defined under static conditions, the maximum tensile stress and the bending moment can be expressed in terms of the displacement. Some authors have developed the dynamic response of the test specimens even further by superposing several modes [20], [21]. In the case of a notched beam with a short time to fracture subjected to one-point bending tests, the latter authors established that the beam model is not relevant. The response of the specimen was therefore modelled using finite element methods [22], [23], where the eigenmodes were determined using a 2-D model for the beam [24]. The maintenance of contact and loss of contact at the supporting points has also been studied experimentally in [25].

- If the reaction of the supports remains equal to zero until complete fracture, we will call this dynamic response an impulsive response since duration is short compared to the time needed for a wave to propagate to the specimen ends and return back to the impact point. This case is met in the Hopkinson Bar bending test on quasibrittle materials which are known to fracture at very low strain levels and with very little energy dissipation. 
- Spall response : duration comparable to or less than the transit time through the specimen transverse sizes. In this case, the superposition of two waves in the specimen thickness, incident compressive pulse and reflected tensile pulse, sets material in tension and causes damage leading to spallation [26].

The present study is concentrating on the impulsive response of specimens of quasi-brittle materials with no notches [27]. To be able to analyse the results properly, it is necessary to perform a transient dynamic analysis on the structure. The approach proposed in this paper for analysing and interpreting these tests takes into account the fact that no information is transmitted back from the support to the impact point before complete fracture occurs.

In section 2, after describing the experimental set-up used and the specificities of the test, a theoretical modelling procedure is presented for determining the transient elastic response of the specimen. The novel approach presented here is applied in section 3 to an example in which an unnotched clay brick specimen was tested and the dynamic mechanical strength is deduced. In section 4, comparisons with classical approaches and one dimensional and twodimensional finite element simulations are performed. These simulations are used to check, on the one hand, the validity of a one-dimensional beam modelling approach and, on the other hand, the accuracy of the proposed model. Section 5 is the conclusion where we suggest possible future research.

\section{A model for dynamic bending test on quasi-brittle materials interpretation}

\subsection{The experimental set-up and specificities of the test}

A scheme of the set-up used in the present study is shown in Fig. 1. The two output (or transmission) bars were used to support the specimen, and the load was applied via the input (or incident) bar. The bending test set-up and the specimen can be seen in the photographs in Fig. 2. The strain waves in the bars were measured using resistance gauges. The impact velocity was measured via photoelectric cells. The characteristics of the bars are specified in table 1 .

\begin{tabular}{ll}
\hline Length of the striker & $L_{I}=1.25 \mathrm{~m}$ \\
Length of the incident bar & $L_{B}=3 \mathrm{~m}$ \\
Diameter of the bars & $\phi_{B}=40 \times 10^{-3} \mathrm{~m}$ \\
Cross-section of the bars & $S_{B}=12.57 \times 10^{-4} \mathrm{~m}^{2}$ \\
Young's modulus & $E_{B}=74 \mathrm{GPa}$ \\
Density & $\rho_{B}=2800 \mathrm{~kg} \mathrm{~m}^{-3}$ \\
Uniaxial wave celerity & $C_{B}=5140 \mathrm{~m} \mathrm{~s}^{-1}$ \\
Impedance & $Z_{B}=18090 \mathrm{~kg} \mathrm{~s}^{-1}$ \\
\hline
\end{tabular}

Table 1: Characteristics of the aluminium Hopkinson bars

The characteristics of the specimen tested are specified in table 2; the Young's modulus was determined using quasi-static three-point bending tests and a Poisson's ratio of 0.2 was assumed.

\begin{tabular}{ll}
\hline Length & $L_{s}=22 \times 10^{-2} \mathrm{~m}$ \\
Thickness & $a=1.7 \times 10^{-2} \mathrm{~m}$ \\
Width & $b=6.5 \times 10^{-2} \mathrm{~m}$ \\
Young's modulus & $E=7 \mathrm{GPa}$ \\
Density & $\rho=1400 \mathrm{~kg} \mathrm{~m}^{-3}$ \\
Distance between supports & $L=20 \times 10^{-2} \mathrm{~m}$ \\
\hline
\end{tabular}

Table 2: Characteristics of the test specimen

The velocity $V_{e}(t)$ and the force $F_{e}(t)$ at the contact point between the incident bar and the test specimen were determined using the following classical formulae [5]:

$$
V_{e}(t)=-C_{B}\left(\varepsilon_{i}(t)-\varepsilon_{r}(t)\right)
$$




$$
F_{e}(t)=-S_{B} E_{B}\left(\varepsilon_{i}(t)+\varepsilon_{r}(t)\right)
$$

where $\varepsilon_{i}$ and $\varepsilon_{r}$ are the incident and reflected wave known at the bar-specimen interface.

Bending test interpretations are feasible if it is possible to deduce the stresses and strains at fracture from the experimental measurements (i.e., the impact force and the impact velocity). It is therefore necessary to have an appropriate modelling technique, in terms of both time and space, to describe the kinematics of the test specimen and to assess the internal stresses.

A reference time $T_{R}$ is introduced, and defined as the effective duration of the bending test. This parameter corresponds to the time elapsing between the beginning of the impact and the fracture of the specimen. The force exerted by the incident bar on the test specimen generates a compression wave at the impact point, which propagates through the material. The term initial response of the solid was used to denote the mechanical state induced by the propagation of the compression waves followed by the tension waves resulting from a reflection at the opposite face of the specimen. Since these waves propagate at the primary waves'celerity $C_{p}$, the characteristic duration of this phase can be taken to be equal to that of the wave propagation across the width of the solid:

$$
T_{T}=\frac{a}{C_{p}}
$$

With durations longer than $T_{T}$, the waves will be reflected and diffracted several times. Then it is reasonable to assume that the motions can be studied on the scale of the structure, and that a model for this structure can be used for this purpose [1]. The configuration of the specimen (table 2) made it possible to use a beam modelling method. Under static conditions, beam modelling gives an excellent assessment of the stresses and strains when the beam is elongated, whereas under dynamic conditions, the condition which has to be met focuses on the wavelength of the transverse motion of the beam. A specimen can be treated as a beam only if the following two conditions are met:

- the characteristic duration of the process under investigation is sufficiently large compared to the time taken by the wave to propagate across the width of the specimen

- the wavelength of these transverse displacements $\lambda$ is large enough in comparison with the radius of giration of the cross section $r=\sqrt{I / S}$ where $S$ is the cross-sectional area and $I$ is the second moment of the cross-sectional area.

If the duration of the bending test $T_{R}$ is short in comparison with the time $T_{C}$ taken by the motions generated by the impact to propagate to the specimen ends and return back to impact point, there will be no influence of the supports on the fracture. In this case, we will have the same problem as that involving a beam with an infinite length. The infinite length assumption is based on the fact that no information is transmitted back from the supports before fracture occurs. A minoration of the time $T_{C}$ can be obtained considering that the bending waves cannot propagate faster than the primary waves:

$$
T_{C}>\frac{L}{C_{p}}
$$

The main characteristics of wave propagation in the specimen are specified in table 3.

\begin{tabular}{ll}
\hline Spall characteristic time & $T_{T}=7.2 \mu \mathrm{s}$ \\
Radius of gyration & $r=4.9 \times 10^{-3} \mathrm{~m}$ \\
Support reaction characteristic time & $T_{C}>\frac{L}{C_{p}}=85 \mu \mathrm{s}$. \\
\hline
\end{tabular}

Table 3: Wave propagation characteristics of the test specimen

The recorded signals shown in Fig. 3 were obtained by performing a dynamic bending test on a baked clay brick. The change in the reflected wave shape is a clear evidence that significant physical damage occurs within a very a short time interval (surrounded area in Fig. 3). This is the main particularity of tests on quasi-brittle materials: the duration 
of the incident pulse $(\approx 500 \mu s)$, which depends on the length of the striker, easily suffices for fracture to occur (these tests could be carried out with shorter bars and a shorter striker). No reaction was present in the transmission bars. In other words, it was as if there were no transmission bars.

\subsection{The long beam model for bending test interpretation}

Dynamic tests do not consist like quasi-static tests in imposing a known, controlled force or displacement on a test specimen. With the SHPB set-up, the loading results from dynamic interactions between the test specimen and the incident bar. The feasibility of the test depends on the existence of an impedance match between the bars and the specimen tested. The relation between $V_{e}$ and $F_{e}$ (the velocity and the force at the end of the incident bar) determines the relation between the incident and reflected waves ( equations (1) and (2)). In bending tests, the elastic behaviour of the test specimens, which relates $V_{e}$ to $F_{e}$, determines the reflection of the incident wave and hence the loading conditions. The model for the bending can be used to determine the strength of the material and other intrinsic characteristics from the experimental data.

To illustrate how an interaction between a bar and a specimen generates a load, a model was specifically developed to deal with the case of quasi-brittle materials. The framework used for this purpose will be that of a beam subjected to an impact, which is simulated by a time-dependent point force (force $F_{e}(t)$ at $x=0$ ). The simplest beam model was introduced by Bernoulli. In this model for a beam subjected to bending loads, the distortions resulting from shear forces are neglected. The cross-sections are always plane and perpendicular to the beam axis; the rotation of a cross-section is therefore equal to the slope of the deformed beam (Fig. 4). Under these assumptions, the equation of motion $w(x, t)$ can be written as follows, neglecting the rotary inertia but not the translation inertia :

$$
\frac{\partial^{4} w}{\partial x^{4}}+4 \alpha^{4} \frac{\partial^{2} w}{\partial t^{2}}=0 \quad \text { where } \quad 4 \alpha^{4}=\frac{\rho S}{E I}
$$

In this equation $\left.x \in] 0, \frac{L}{2}\right]$. Since we suppose that $T_{R}<<T_{C}$, the events occurring in the centre of the beam will not be influenced by the supports, just as if the beam was of infinite length. The x-range becomes $x \in] 0$, $+\infty$ [ with the following boundary conditions at the impact point and at the far end of the beam:

$$
\frac{\partial w}{\partial x}(0, t)=0 \quad, \quad \frac{\partial^{3} w}{\partial x^{3}}(0, t)=\frac{F_{e}(t)}{2 E I} \quad \text { and } \quad w(+\infty, t)=0
$$

The impulse response obtained when the impact force is a Dirac function $F_{e}(t)=\delta(t)$ was first established by Ditkine V. and Proudnikov A. [28]. In order to make the best possible use of the experimental data, it is proposed to take a slightly different problem: that of a long beam, where two boundary conditions at $x=0$, the displacement $U_{e}$ and the force $F_{e}$, are known, based on the measurements performed, and we have to determine the other two, namely the rotation $\psi(0, t)$ and the bending moment $M(0, t)$ (see Fig. 5). This new problem takes into account the observed occurence of crack initiation and propagation under the impact point. It contains the previous problem as a particular case $(\psi(0, t)=0)$ but is more general since it remains valid after the onset of a first centered crack. As a consequence, it could be used to describe the cracking behaviour of the specimen. The relations at the impact point are modified by cracking as:

$$
w(0, t)=U_{e}(t) \quad, \quad \frac{\partial w}{\partial x}(0, t)=\psi(0, t) \quad, \quad \frac{\partial^{2} w}{\partial x^{2}}(0, t)=-\frac{M(0, t)}{E I} \quad, \quad \frac{\partial^{3} w}{\partial x^{3}}(0, t)=\frac{F_{e}(t)}{2 E I}
$$

$U_{e}(t)$ and $F_{e}(t)$ are known (measured) functions, and $\psi(0, t)$ and $M(0, t)$ are unknown functions. At the far end of the beam, we take $w(+\infty, t)=0$ (along with all the corresponding time derivatives).

The transient dynamic elastic response of the test specimen can be determined from the experimental data (the details of the application of operational calculus are given in Appendix):

$$
w(x, t)=\int_{0}^{t} G_{1}(t-\tau) \Omega_{1}(x, \tau) d \tau-\int_{0}^{t}\left(G_{1}(t-\tau)+G_{2}(t-\tau)\right) \Omega_{2}(x, \tau) d \tau
$$

where

$$
G_{1}(t)=\int_{0}^{t} \frac{V_{e}(\tau)}{\sqrt{\pi(t-\tau)}} d \tau \quad \text { and } \quad G_{2}(t)=\int_{0}^{t} \frac{F_{e}(\tau)}{4 E I \alpha^{3}} d \tau
$$




$$
\Omega_{1}(x, t)=\frac{1}{\sqrt{\pi t}} \cos \left(\frac{\alpha^{2} x^{2}}{2 t}\right) \text { and } \Omega_{2}(x, t)=\frac{1}{\sqrt{\pi t}} \sin \left(\frac{\alpha^{2} x^{2}}{2 t}\right)
$$

From which the following can be deduced:

- Rotation and bending moment at $x=0$

$$
\begin{gathered}
\psi(0, t)=-2 \alpha \int_{0}^{t} \frac{V_{e}(\tau)}{\sqrt{\pi(t-\tau)}} d \tau+\frac{1}{4 E I \alpha^{2}} \int_{0}^{t} F_{e}(\tau) d \tau \\
M(0, t)=\frac{1}{2 \alpha} \int_{0}^{t} \frac{F_{e}(\tau)}{\sqrt{\pi(t-\tau)}} d \tau-2 E I \alpha^{2} V_{e}(t)
\end{gathered}
$$

- If we take the rotation at the origin to be zero prior to the fracture process, we obtain the following relation for the interaction imposed at the end of the incident bar between the force and the velocity during the elastic phase:

$$
V_{e}(t)=\frac{1}{2 \eta} \int_{0}^{t} \frac{F_{e}(\tau)}{\sqrt{\pi(t-\tau)}} d \tau \text { where } \quad \eta=4 E I \alpha^{3} \quad \text { and } \quad 4 \alpha^{4}=\frac{\rho S}{E I}
$$

and the relation between the incident and reflected waves:

$$
\varepsilon_{r}(t)=\varepsilon_{i}(t)-\int_{0}^{t} \frac{2 \varepsilon_{i}(\tau)}{\sqrt{\tau_{f}}}\left(\frac{1}{\sqrt{\pi(t-\tau)}}-\frac{1}{\sqrt{\tau_{f}}} e^{\frac{t-\tau}{\tau_{f}}} \operatorname{erf} c\left(\sqrt{\frac{t-\tau}{\tau_{f}}}\right)\right) d \tau
$$

where $\operatorname{erfc}(t)=\frac{2}{\sqrt{\pi}} \int_{t}^{\infty} e^{-\tau^{2}} d \tau \quad$ denotes the complementary error function and $\tau_{f}=\left(\frac{2 \eta}{Z_{B}}\right)^{2}$.

\section{Application of the long beam model to processing experimental data}

Analysing the results of the test requires, in a first stage, subtracting the incident and the reflected waves in order to determine the velocity at the impact point (equation (1)), and then adding these two waves to determine the force exerted at the same point (equation 2)). Since the strains are measured in the middle of the incident bar, a wave transport has to be expressed in terms of time, taking the dispersion into account. The two waves have to be closely synchronised in order to accurately describe the whole test range. Once the velocity and the force are known at the impact point, rotation and bending moment are deduced from the equations (11) and (12).

\subsection{Time shifting of the waves based on an elastic response}

In practice, modelling transport time by taking only the distances to the specimen faces and the celerity of the waves into account is not a sufficiently accurate approach. In the case of bending tests, the shape of the end of the input bar can, for exemple, give rise to a lack of accuracy. The best way of synchronising the waves is to simulate the reflection of the incident wave front in order to determine the onset of the reflected wave measured. Although this modelling method has already been applied to compression tests [6], the long beam model is proposed as an appropriate one for processing bending tests data. During the first few instants after the onset of the loading, the behaviour of the specimen was assumed to remain elastic, and the relation (14) between the incident and reflected waves could therefore be used. This relation was used to simulate the elastic response of the specimen. From the incident wave measurements, it was possible to compute the reflected wave corresponding to an elastic response of the specimen and to exactly time the onset of the reflected wave measured. The incident and reflected waves measured and the reflected wave predicted by the elastic response using the relation (14), all of which were time shifted, are given in Fig. 6. 


\subsection{Non dissipative behaviour}

Fig. 7a and Fig. 7b give the velocities and forces obtained using relations (1) and (2). On the one hand, the wave measurements recorded during the tests were used and on the other hand, the incident wave and the reflected wave measurements corresponding to the elastic response of the specimen were used. It can be seen from these figures that when $t<40 \mu \mathrm{s}$, the curves based on the measured signals (time shifted) and those based on the elastic response either coincided or showed excellent agreement. This result confirms the assumption that the behaviour of the beam at the beginning of the test was purely elastic. The onset of a non linear behaviour, which was assumed to be concomitant with the cracking process, can be seen from the separation of the curves $\left(T_{R} \approx 40 \mu \mathrm{s}\right)$. During the elastic phase, from (12) and taking the relation (13) into account, the bending moment is given by:

$$
M(0, t)=\frac{1}{4 \alpha} \int_{0}^{t} \frac{F_{e}(\tau)}{\sqrt{\pi(t-\tau)}} d \tau=2 E I \alpha^{2} V_{e}(t)
$$

which yields the maximum through-the-thickness tensile stress:

$$
\sigma(0, t)=\frac{a M(0, t)}{2 I}=\frac{a}{8 I \alpha} \int_{0}^{t} \frac{F_{e}(\tau)}{\sqrt{\pi(t-\tau)}} d \tau=a E \alpha^{2} V_{e}(t)
$$

and the maximum through-the-thickness strain rate:

$$
\dot{\varepsilon}=a \alpha^{2} \frac{d V_{e}}{d t}
$$

It can be noticed that the moment and the maximum tensile stress are proportional to the velocity at the impact point, and not to the displacement. It is also worth noting that the strain rate depends on the acceleration at the impact point and not on the velocity. The curves giving the impact velocity $V_{e}$ as a function of time Fig. 7a can be seen to show a quasi-linear part during the elastic phase. The strain rate associated with this phase, which was obtained from relation (17) is denoted $\dot{\varepsilon}^{c}$ and takes the value $\dot{\varepsilon}^{c}=250 s^{-1}$ in the reported test. With our experimental set-up, the magnitude of the strain rate applied to quasi-brittle materials ranges typically from $20 s^{-1}$ to $500 s^{-1}$.

\subsection{Dissipative behaviour}

When cracking occurs, the "zero rotation at the origin" condition no longer applies. This rotation and the residual moment can be calculated in the case of partial fracture of the specimen by applying the measurements obtained to relations (11) and (12). In the tests, cracks occurred under the impact force. At $t>T_{R} \approx 40 \mu \mathrm{s}$, non linear phenomena were therefore presumably present only in the central cross section of the specimen.

The information obtained about the evolution of the moment with the crack opening $2 \psi$, which is shown in Fig. 8, makes it possible:

- to detect more closely than in Fig. 7a and Fig. 7b the initiation of the cracking process, and hence the strength of the tested material. The elastic phase of the test was expected to correspond to a straight vertical segment, since the crack opening remains zero, whereas the moment is on the increase;

- to define a law governing the relation between the moment and the opening of the crack.

The mechanical strength of the brick material tested in this study is presented in table 4. As explained above, the dynamic values were deduced from the changes with time in the moment (Fig. 8), since in the context of elastic behaviour, $\sigma=\frac{a M}{2 I}$. The static value was obtained using a classical three-point bending test. It can be noticed an increase of the mechanical strength in the dynamic situation compared to the static one. 


\begin{tabular}{ccc} 
& static & dynamic \\
\hline$\dot{\varepsilon}^{c}$ & $0.005 s^{-1}$ & $250 s^{-1}$ \\
$\sigma^{e}$ & $7.7 \mathrm{MPa}$ & $33 \mathrm{MPa}$ \\
\hline
\end{tabular}

Table 4: Mechanical strength of the brick specimen

\section{Discussion on the validity of the long beam model}

Since the real specimen is a three-dimensional object, we must discuss the validity of a one-dimensional theory to model accurately the short-time response of the sample. For an imposed impact in the middle of the beam, there is a finite time, greater than $\frac{L}{C_{p}}$, during which the smallest wavelength will not return back to the point of application of the impulse. The proposed long-beam model is an Euler-Bernouilli beam model which predicts an approaching infinite wave speed for an infinitesimal wavelength. The Timoshenko beam model taking rotary inertia and shear into account is usually considered as an improvement since the wave speeds remain bounded. But for a given input load, the small wavelength components have small amplitudes. With an Euler-bernouilli model, if we cannot say that, during the studied short-time, some spurious high frequency waves will not be reflected to the impact point, we can rather suppose that what will be reflected is negligible. Nevertheless this assumption has to be investigated.

A two-dimensional reference numerical elastic solution is used to check the legitimacy of using one-dimensional beam models. The test case is that of a specimen tested to a force ramp. Even if dynamic tests using Hopkinson bar do not consist in imposing a known controlled force on a test specimen but results from dynamic interactions between the test specimen and the end of the incident bar, a unit force ramp (i.e. $\left.F_{e}(t)=t\right)$ was used. This simple loading was only chosen because:

- it was suitable for the comparisons between explicit methods and the finite element simulations;

- the finite element simulations were easier to perform than in the real case where it could be necessary to model the whole experimental device and not only the specimen;

- it allowed to calculate an analytical solution with different classical models.

The FEM simulations were performed using the finite element code Cast $3 \mathrm{M}^{1}$ developed at the French Atomic Energy Commission's Department of Mechanics and Technology (DMT) [29]. The simulations were carried out in 2-D situations, since the results obtained in 3-D simulations with coarse mesh were equivalent. The meshing elements used were quadrilaterals with 8 nodes and a quadratic interpolation. The simulations were performed under plane strain state assumptions. Time resolution was performed using the implicit Newmark scheme. In view of the symmetry of the problem, only half of the specimen was meshed. The mesh was refined up to convergence of the static reference calculations. The regular meshing procedure adopted included 7295 nodes and 2340 elements (grid of $20 \times 117$ ). 1-D simulations were also carried out using Euler-Bernoulli beam elements and Timoshenko beam elements meshed using the same axial mesh discretisation procedure as above.

To check the improvement over standard approaches, the result obtained by the long beam model was also compared with those predicted by a quasi-static approach, by a modelling of the beam using a single degree of freedom system (SDOF) or using a modal superposition method :

- quasi-static state beam model:

The stresses and strains can be written quite simply. The relation between the force and the displacement at the end of the incident bar is:

$$
U_{e}=\frac{F_{e}}{k_{e}} \quad \text { where } \quad k_{e}=\frac{48 E I}{L^{3}}
$$

\footnotetext{
${ }^{1}$ Cast3M, http://www-cast3m.cea.fr
} 
and leads to

$$
\sigma(t)=\frac{a L t}{8 I}
$$

\section{- SDOF beam model:}

The previous model can be improved by taking the inertial forces into account. This model based on Rayleigh's method consists in approximating the beam via a single degree of freedom (SDOF) system, assuming the shape of the dynamically deformed beam to be similar to that of the quasi-static deformed beam.

$$
m_{e} \ddot{U}_{e}+k_{e} U_{e}=F_{e} \quad \text { where } \quad m_{e}=\frac{17}{35} \rho S L
$$

The interaction relation imposed at the center of the beam between the force and the displacement is:

$$
U_{e}(t)=\frac{1}{m_{e} \omega_{e}} \int_{0}^{t} F_{e}(\tau) \sin \left(\omega_{e}(t-\tau)\right) d \tau \quad \text { where } \quad \omega_{e}=\sqrt{\frac{k_{e}}{m_{e}}}
$$

and leads to

$$
\sigma(t)=\frac{a L}{8 I}\left(t-\frac{1}{\omega_{e}} \sin \left(\omega_{e} t\right)\right)=\frac{6 a E}{m_{e} \omega_{e}^{2} L^{2}}\left(t-\frac{1}{\omega_{e}} \sin \left(\omega_{e} t\right)\right)
$$

- modal superposition analysis:

The motion of the beam is described in this model by superposing vibration modes. The interaction relation imposed at the center of the beam between the force and the displacement is:

$$
U_{e}(t)=\sum_{j=1}^{\infty} \frac{1}{m_{j} \omega_{j}} \int_{0}^{t} F_{e}(\tau) \sin \left(\omega_{j}(t-\tau)\right) d \tau
$$

and leads to

$$
\sigma(t)=\frac{a E}{2} \sum_{j=1}^{n} \frac{(2 j-1)^{2} \pi^{2}}{L^{2}} f_{j}(t) \quad \text { where } \quad f_{j}=\frac{\omega_{j} t-\sin \left(\omega_{j} t\right)}{m_{j} \omega_{j}^{3}}
$$

- long beam model

Taking into account $F_{e}(t)=t$ in the expression (16) of the maximum stress leads to:

$$
\sigma(t)=\frac{a}{6 I \alpha \sqrt{\pi}} t^{\frac{3}{2}}
$$

Since the main variable of interest is the mechanical strength, we consider the maximum stress to compare the different models. The finite element simulations had also given the time required to obtain a non nil reaction at the supports $(\approx 300 \mu s)$. As assumed in the section 2.1 the duration of analysis was choosen shorter than this time $(100 \mu s)$.

Results presented in Fig. 9 and in Fig.10 showed:

- a good agreement between both the finite element simulations performed ( 2-D simulation and 1-D simulation using beam elements), which shows the relevance of using a one-dimensional beam model,

- no amelioration produced by the use of a Timoshenko beam model,

- a good agreement between the long beam model predictions and those of the finite element simulations,

- a wrong response of the quasi-static approach what was expected as the approach by a SDOF system in a less obvious manner a priori,

- fairly satisfactory results for the modal superposition with a relatively large number of modes (around thirty). However it remains less accurate than the long beam model.

From the discussion above, it is clear that the long beam model is remarkably accurate compared to the twodimensional and to classical approaches provided that the time response studied is short. 


\section{Conclusion}

The approach presented in this paper can be used to interpret the results of dynamic bending tests on quasi-brittle material specimens: this was not possible with the classical methods available so far because failure occurs very early, at very low strain levels. The method developed specifically for this purpose gives accurate and reliable results. As in all tests involving SHPB, with which fracture occurs at very low strains, it is necessary to carefully apply accurate time shifting methods to the wave measurements. The key element of this approach is the long beam analytical model used to simulate the transient elastic response of the tested specimens. The limitations of the method presented here are due to the assumptions required to obtain the analytical solution, i.e., in this case, those focusing on the dimensions of the specimen and the crack initiation time, which fit the assumptions of the "long beam model". One of the main results obtained here was that it is possible with this method to determine accurately the instant at which cracks are initiated and to define the mechanical strength of a material by drawing up a moment versus crack opening curve, from which it should be possible in the future to predict the energy dissipated when bending failure occurs.

The range of potential applications is wider than what this study might seem to indicate, since it could include many geomaterials and construction components. In some of these situations, a similar approach using a Timoshenko beam model must be introduced because the distortions resulting from shear forces are not neglectible.

\section{Acknowledgments}

The authors would like to thank the French National Research Agency (ANR) for supporting this research (in the framework of the VULCAIN ANR-07-PGCU project)

\section{References}

[1] Toutlemonde F, Gary G. (2008) Dynamic behavior of concrete : experimental aspects, in Dynamic behavior of concrete and seismic engineering, Mazars J., Millard A eds ISTE London.

[2] Toutlemonde F, Gary G. Dynamic behavior of concrete: experimental aspects. In Dynamic Behavior of Concrete and Seismic Engineering. Mazars J, Millard A (eds). Wiley: ISTE london, 2009; 1-54.

[3] Bailly P. Présentation d'un banc d'essai dynamique de matériaux et de structures: exemple d'application à l'étude de structures en mortier. Materials and Structures 1994; 27,(172),452-459.

[4] Hopkinson B. A method of measuring the pressure produced in the detonation of high explosives or by the impact of bullets. Philos. Trans. R. Soc. (London) A 1914; 213,437-456.

[5] Kolsky H. An investigation of the mechanical properties of materials at very high rates of loading Proc. Phys. Soc. (London) B 1949; 62,676700.

[6] Zhao H, Gary G. On the use of SHPB techniques to determine the dynamic behavior of materials in the range of small strains. International Journal of Solids and Structures 1996; 33,(22),3363-3375.

[7] Zielinski A J, Reinhardt H W. Stress behaviour of concrete and mortar at high rates of tensile loading. Cement and Concrete Research 1982; 12,(3),309-319.

[8] Klepaczko J R, Brara A (2001) An experimental method for dynamic tensile testing of concrete by spalling, International Journal of Impact Engineering 2001; 25,(4),387-409.

[9] Lambert D E, Ross C A Strain rate effects on dynamic fracture and strength. International Journal of Impact Engineering 2000; 24,(10), 985-998.

[10] Gary G, Bailly P (1998) Behaviour of a quasi-brittle material at high strain rate. Experiment and modelling, European Journal of Mechanics A - Solids 1998; 17,(3), 403-420.

[11] Forquin P, Gary G, Gatuingt F A testing technique for concrete under confinement at high rates of strain. International Journal of Impact Engineering 2008; 35,(6), 425-446.

[12] Yokoyama T, Kishida K. A novel impact three-point bend test method for determining dynamic fracture-initiation toughness. Experimental Mechanics 1989; 29,(2),188-194.

[13] Yokoyama T. Determination of dynamic fracture-initiation toughness using a novel impact bend test procedure. Journal of Pressure Vessel Technology 1993; 115,(4),389-397.

[14] Rizal S, Homma H. Dimple fracture under short pulse loading. International Journal of Impact Engineering 2000; 24,(1),69-83.

[15] Ruiz C, Mines R A W. The Hopkinson pressure bar : an alternative to the instrumented pendulum for Charpy tests. International Journal of Fracture 1985; 29,(2),101-109.

[16] Jiang F C, Rohatgi A, Vecchio K S, Cheney J L Response to the discussion by I.V. Rokach of the paper entitled: Analysis of the dynamic responses for a pre-cracked three-point bend specimen. International Journal of Fracture 2005; 131,(3),801-807.

[17] Bacon C, Farm J, Lataillade JL. Dynamic fracture-toughness determined from load-point displacement. Experimental Mechanics 1994; 34,(3),217-223.

[18] Jiang F, Vecchio KS, Rohatgi A. Analysis of modified split Hopkinson pressure bar dynamic fracture test using an inertia model. International Journal of Fracture 2004; 126,(2),143-164. 
[19] Dutton A G, Mines R A W. Analysis of the Hopkinson pressure bar loaded instrumented Charpy test using an inertial modelling technique. International Journal of Fracture 1991; 51,(2),187-206.

[20] Sahraoui S, Lataillade J L. Analysis of load oscillations in instrumented impact testing. Engineering Fracture Mechanics 1998; 60,(4),437446.

[21] Rokach I V Modal approach for processing one- and three-point bend test data for DSIF-time diagram determination. Part I-Theory. Fatigue $\mathcal{E}$ Fracture of Engineering Materials $\mathcal{E}$ Structures 1998; 21,(8),1007-1014.

[22] Guo W G, Li Y L, Liu Y Y. Analytical and experimental determination of dynamic impact stress intensity factor for 40 Cr steel. Theoretical and Applied Fracture Mechanics 1997; 26,(1),29-34.

[23] Weisbrod G, Rittel D. A method for dynamic fracture toughness determination using short beams. International Journal of Fracture 2000; 104,(1),89-103.

[24] Rokach I V. Modal approach for processing one- and three-point bend test data for DSIF-time diagram determination. Part II-Calculations and results. Fatigue $\mathcal{E}$ Fracture of Engineering Materials $\mathcal{E}$ Structures 1998; 21,(8),1015-1026.

[25] Jiang F, Vecchio K S. Experimental investigation of dynamic effects in a two-bar/three-point bend fracture test. Review of Scientific Instruments 2007; 78,(6),063903.

[26] Chevrier P, Klepaczko J R. Spall fracture: Mechanical and microstructural aspects. Engineering Fracture Mechanics 1999; 63,(3), $273-294$.

[27] Richomme S, Bailly P, Delvare F, Mavrot G. Dynamic testing of concrete with SHPB. In Proceedings of the 15th DYMAT Technical Meeting: Crashworthiness and Constitutive relationships for engineering materials Metz, France, 2004; 109-114.

[28] Ditkine V, Proudnikov A. Calcul oprationnel Editions MIR: Moscou, 1979.

[29] CASTEM 2000 (1998) Code de calcul pour l'analyse de structures par la méthode des éléments finis. Guide d'utilisation. Commissariat à l'Energie Atomique, DEN/DM2S/SEMT/LM2S, F-91191 Gif-sur-Yvette, France.

[30] Bhaskar A., Elastic waves in Timoshenko beams: the 'lost and found' of an eigenmode. Proceedings of the Royal Society 2009 , 465, $239-255$.

\section{Appendix: flexural response of a semi-infinite beam}

For the long beam model, the equation of motion is:

$$
\frac{\partial^{4} w}{\partial x^{4}}+4 \alpha^{4} \frac{\partial^{2} w}{\partial t^{2}}=0 \quad \text { where } \quad 4 \alpha^{4}=\frac{\rho S}{E I}
$$

The relations at the impact point are supposed to be:

$$
w(0, t)=U_{e}(t) \quad, \quad \frac{\partial w}{\partial x}(0, t)=\psi(0, t) \quad, \quad \frac{\partial^{2} w}{\partial x^{2}}(0, t)=-\frac{M(0, t)}{E I} \quad, \quad \frac{\partial^{3} w}{\partial x^{3}}(0, t)=\frac{F_{e}(t)}{2 E I}
$$

Using the time Laplace transform, the image of the beam motion that vanishes at infinity has the following form:

$$
\bar{w}(x, s)=e^{-\alpha x \sqrt{s}}\left(\bar{K}_{1} \cos (\alpha x \sqrt{s})+\bar{K}_{2} \sin (\alpha x \sqrt{s})\right)
$$

We then take the following operators and the corresponding time functions:

$$
\begin{array}{cc}
\bar{\Omega}_{1}(x, s)=\frac{e^{-\alpha x \sqrt{s}}}{\sqrt{s}} \cos (\alpha x \sqrt{s}) & , \quad \bar{\Omega}_{2}(x, s)=\frac{e^{-\alpha x \sqrt{s}}}{\sqrt{s}} \sin (\alpha x \sqrt{s}) \\
\Omega_{1}(x, t)=\frac{1}{\sqrt{\pi t}} \cos \left(\frac{\alpha^{2} x^{2}}{2 t}\right) \quad, \quad \Omega_{2}(x, t)=\frac{1}{\sqrt{\pi t}} \sin \left(\frac{\alpha^{2} x^{2}}{2 t}\right)
\end{array}
$$

The image of the response can also be written with these functions:

$$
\bar{w}(x, s)=\sqrt{s}\left(\bar{K}_{1} \bar{\Omega}_{1}+\bar{K}_{2} \bar{\Omega}_{2}\right)
$$

After calculating the partial derivatives with respect to $x$ and taking the boundary conditions into account, we obtain the following expressions:

$$
\begin{gathered}
\bar{w}(0, s)=\bar{K}_{1}=\bar{U}_{e}(s) \\
\frac{\partial \bar{w}}{\partial x}(0, s)=\alpha \sqrt{s}\left(\bar{K}_{2}-\bar{K}_{1}\right)=\bar{\psi}(0, s) \\
\frac{\partial^{2} \bar{w}}{\partial x^{2}}(0, s)=-2 \alpha^{2} s \bar{K}_{2}=\frac{\bar{M}(0, s)}{E I}
\end{gathered}
$$




$$
\frac{\partial^{3} \bar{w}}{\partial x^{3}}(0, s)=2 \alpha^{3} s^{\frac{3}{2}}\left(\bar{K}_{1}+\bar{K}_{2}\right)=\frac{\bar{F}_{e}(s)}{2 E I}
$$

from which it can be deduced that

$$
\bar{K}_{1}(s)=\bar{U}_{e} \quad, \quad \bar{K}_{2}(s)=\frac{\bar{F}_{e}}{4 E I \alpha^{3} s^{\frac{3}{2}}}-\bar{U}_{e}
$$

In the operational space, the motion is:

$$
\bar{w}(x, s)=\sqrt{s}\left(\bar{U}_{e} \bar{\Omega}_{1}+\left(-\bar{U}_{e}+\frac{\bar{F}_{e}}{\eta s^{\frac{3}{2}}}\right)\right) \bar{\Omega}_{2} \quad \text { where } \quad \eta=4 E I \alpha^{3}
$$

The transient dynamic elastic response of the test specimen can be determined from the experimental data. :

$$
w(x, t)=\int_{0}^{t} G_{1}(t-\tau) \Omega_{1}(x, \tau) d \tau-\int_{0}^{t}\left(G_{1}(t-\tau)+G_{2}(t-\tau)\right) \Omega_{2}(x, \tau) d \tau
$$

where

$$
G_{1}(t)=\int_{0}^{t} \frac{V_{e}(\tau)}{\sqrt{\pi(t-\tau)}} d \tau \text { and } G_{2}(t)=\int_{0}^{t} \frac{F_{e}(\tau)}{4 E I \alpha^{3}} d \tau
$$

From which the following can be deduced:

- Rotation and bending moment at $x=0$

- in the operational form

$$
\begin{gathered}
\bar{\psi}(0, s)=\alpha \sqrt{s}\left(\bar{K}_{2}-\bar{K}_{1}\right)=\frac{\bar{F}_{e}(s)}{4 E I \alpha^{2} s}-2 \alpha \sqrt{s} \bar{U}_{e}(s)=\frac{\bar{F}_{e}(s)}{4 E I \alpha^{2} s}-2 \alpha \frac{\bar{V}_{e}(s)}{\sqrt{s}} \\
\bar{M}(0, s)=2 E I \alpha^{2} s \bar{K}_{2}=-2 E I \alpha^{2} s \bar{U}_{e}(s)+\frac{\bar{F}_{e}(s)}{2 \alpha \sqrt{s}}=-2 E I \alpha^{2} \bar{V}_{e}(s)+\frac{\bar{F}_{e}(s)}{2 \alpha \sqrt{s}}
\end{gathered}
$$

- in the time domain

$$
\begin{gathered}
\psi(0, t)=-2 \alpha \int_{0}^{t} \frac{V_{e}(\tau)}{\sqrt{\pi(t-\tau)}} d \tau+\frac{1}{4 E I \alpha^{2}} \int_{0}^{t} F_{e}(\tau) d \tau \\
M(0, t)=\frac{1}{2 \alpha} \int_{0}^{t} \frac{F_{e}(\tau)}{\sqrt{\pi(t-\tau)}} d \tau-2 E I \alpha^{2} V_{e}(t)
\end{gathered}
$$

- If we take the rotation at the origin to be zero prior to the fracture process, we obtain:

$$
\bar{K}_{1}(s)=\bar{K}_{2}(s)
$$

and the following relation for the interaction imposed at the end of the incident bar between the force and the velocity during the elastic phase:

- in the operational form

$$
\bar{V}_{e}(s)=\frac{1}{2 \eta \sqrt{s}} \bar{F}_{e}(s)
$$

- in the time domain

$$
V_{e}(t)=\frac{1}{2 \eta} \int_{0}^{t} \frac{F_{e}(\tau)}{\sqrt{\pi(t-\tau)}} d \tau \quad \text { where } \quad \eta=4 E I \alpha^{3} \quad \text { and } \quad 4 \alpha^{4}=\frac{\rho S}{E I}
$$


and the relation between the incident and reflected waves:

- in the operational form

$$
\bar{\varepsilon}_{r}=\bar{\varepsilon}_{i}\left(\frac{\bar{L}(s)-Z_{B}}{\bar{L}(s)+Z_{B}}\right) \quad \text { where } \quad \bar{L}(s)=\frac{\bar{F}_{e}(s)}{\bar{V}_{e}(s)}=2 \eta \sqrt{s}
$$

or

$$
\bar{\varepsilon}_{r}=\bar{\varepsilon}_{i}\left(\frac{\sqrt{\tau_{f} s}-1}{\sqrt{\tau_{f} s}+1}\right)=\bar{\varepsilon}_{i}\left(1-\frac{2}{\sqrt{\tau_{f} s}+1}\right) \text { where } \tau_{f}=\left(\frac{2 \eta}{Z_{B}}\right)^{2}
$$

- in the time domain

$$
\varepsilon_{r}(t)=\varepsilon_{i}(t)-\int_{0}^{t} \frac{2 \varepsilon_{i}(\tau)}{\sqrt{\tau_{f}}}\left(\frac{1}{\sqrt{\pi(t-\tau)}}-\frac{1}{\sqrt{\tau_{f}}} e^{\frac{t-\tau}{\tau_{f}}} \operatorname{erfc}\left(\sqrt{\frac{t-\tau}{\tau_{f}}}\right)\right) d \tau
$$

where $\operatorname{erfc}(t)=\frac{2}{\sqrt{\pi}} \int_{t}^{\infty} e^{-\tau^{2}} d \tau \quad$ denotes the complementary error function. 


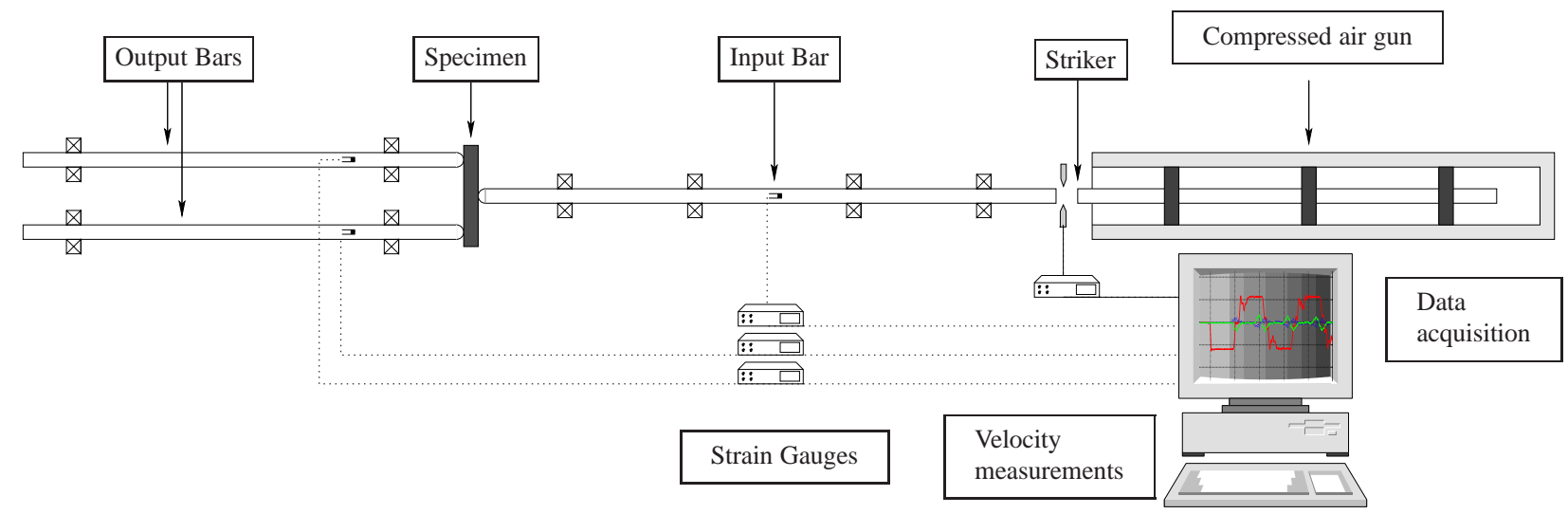

Figure 1: Bending test set-up involving the use of Hopkinson bar. 

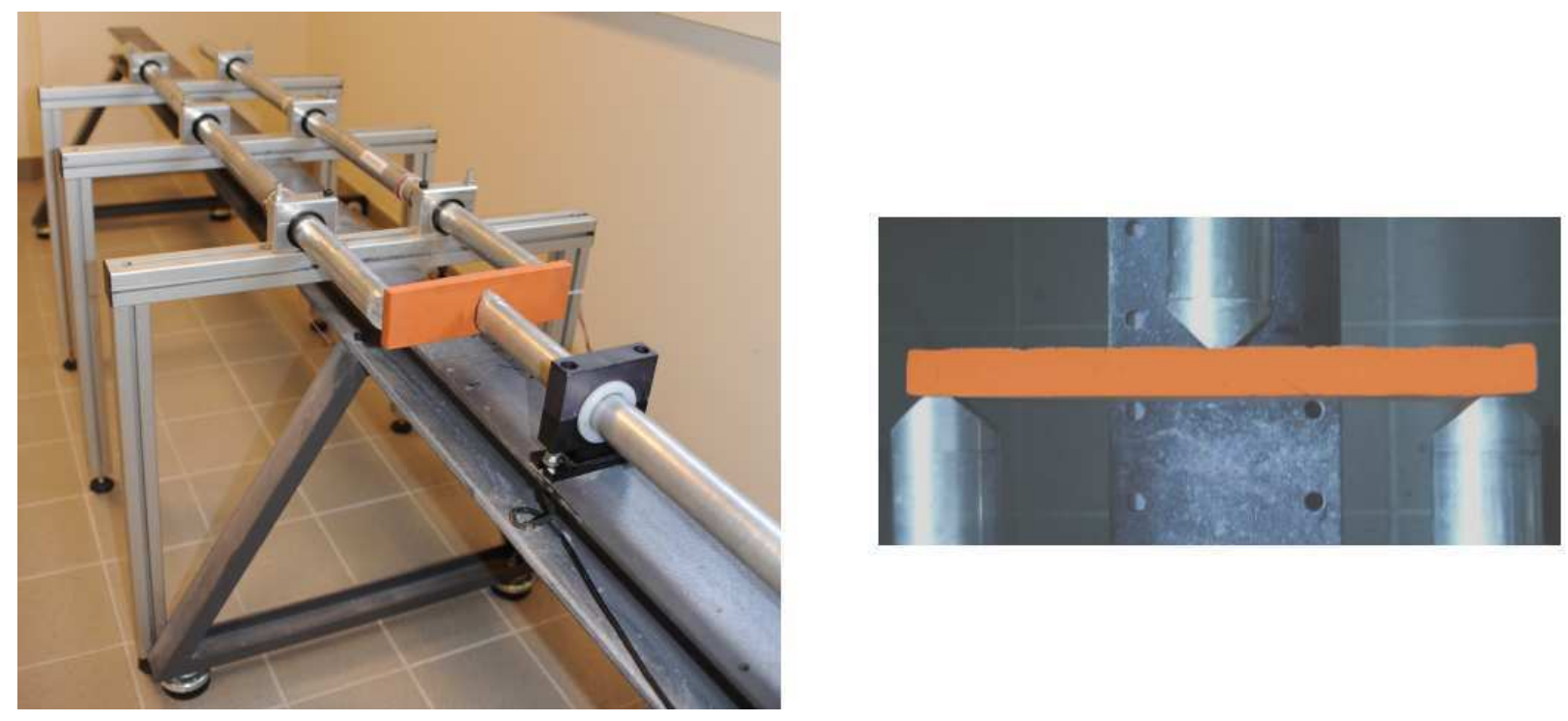

Figure 2: Photographs of the bending test set-up, brick specimen 


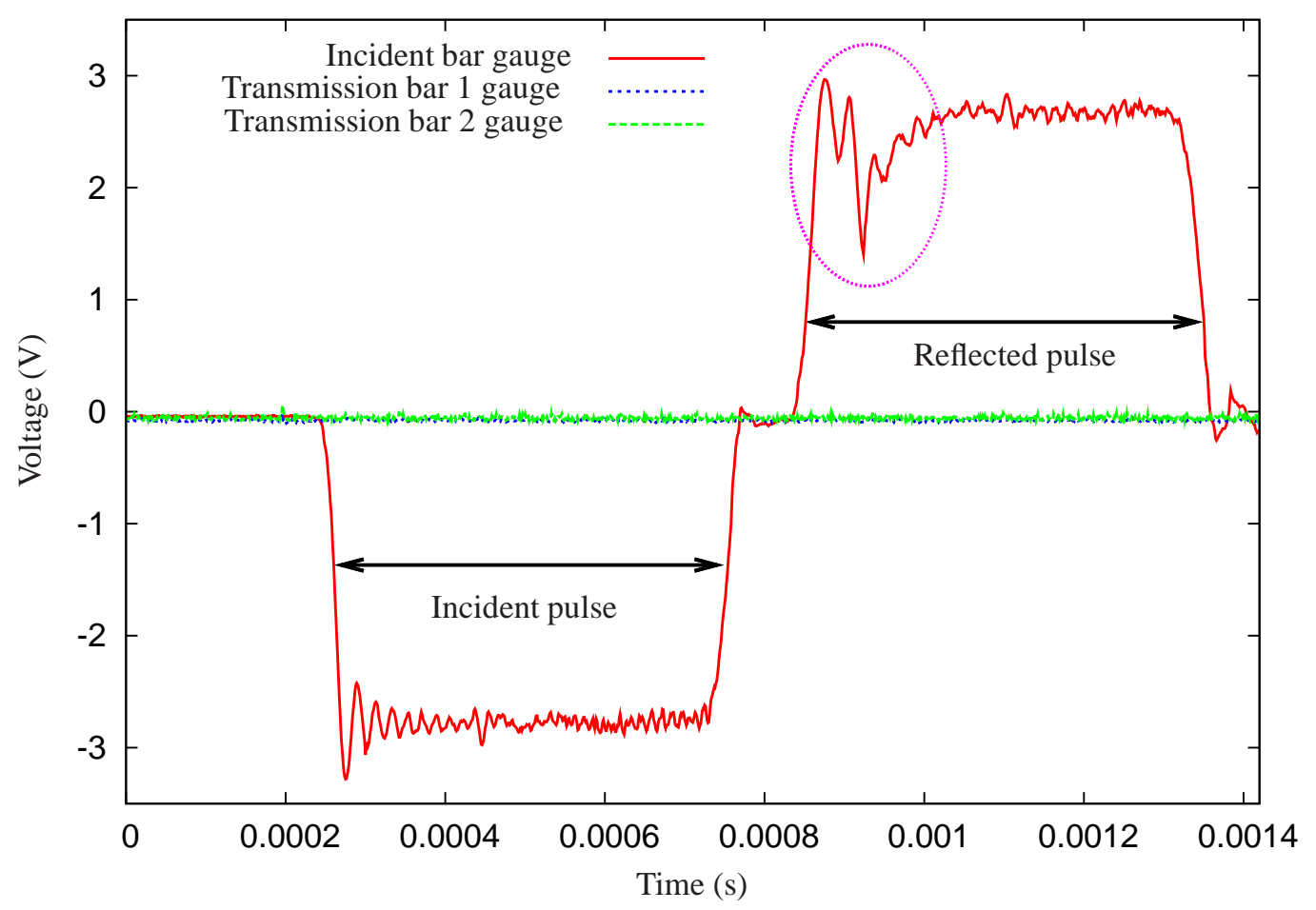

Figure 3: Raw signals measured in the bars 


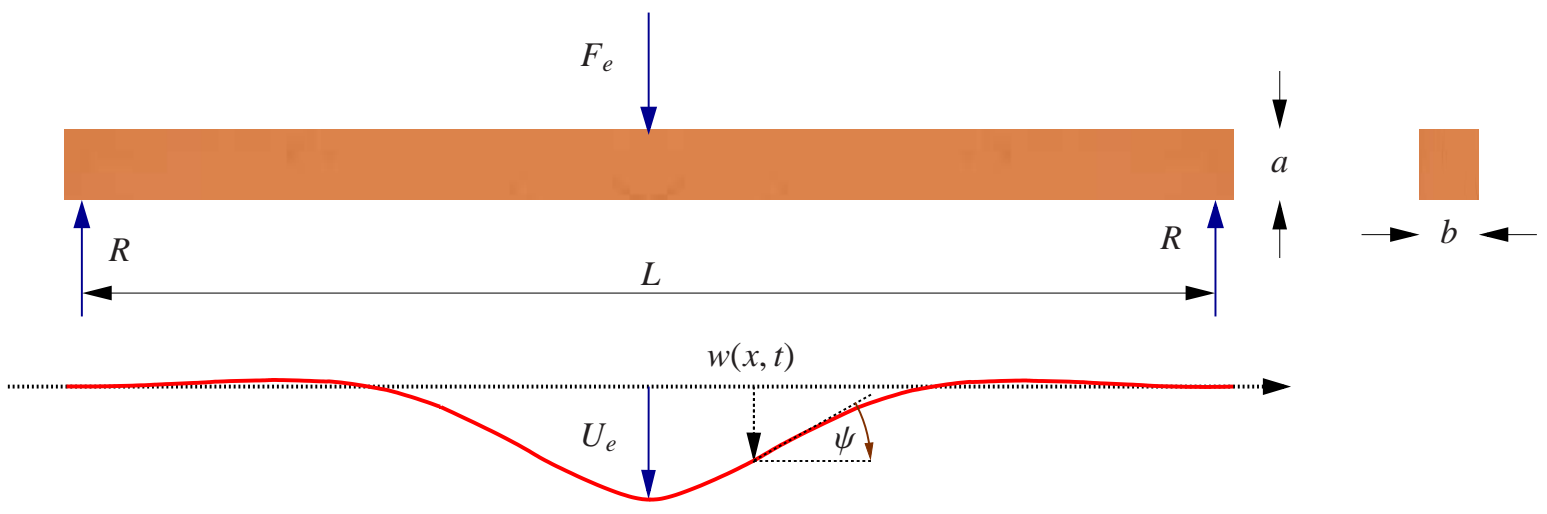

Figure 4: Modelling the specimen like a beam: the transverse displacement $U_{e}$ and the cross section rotation $\psi$ 


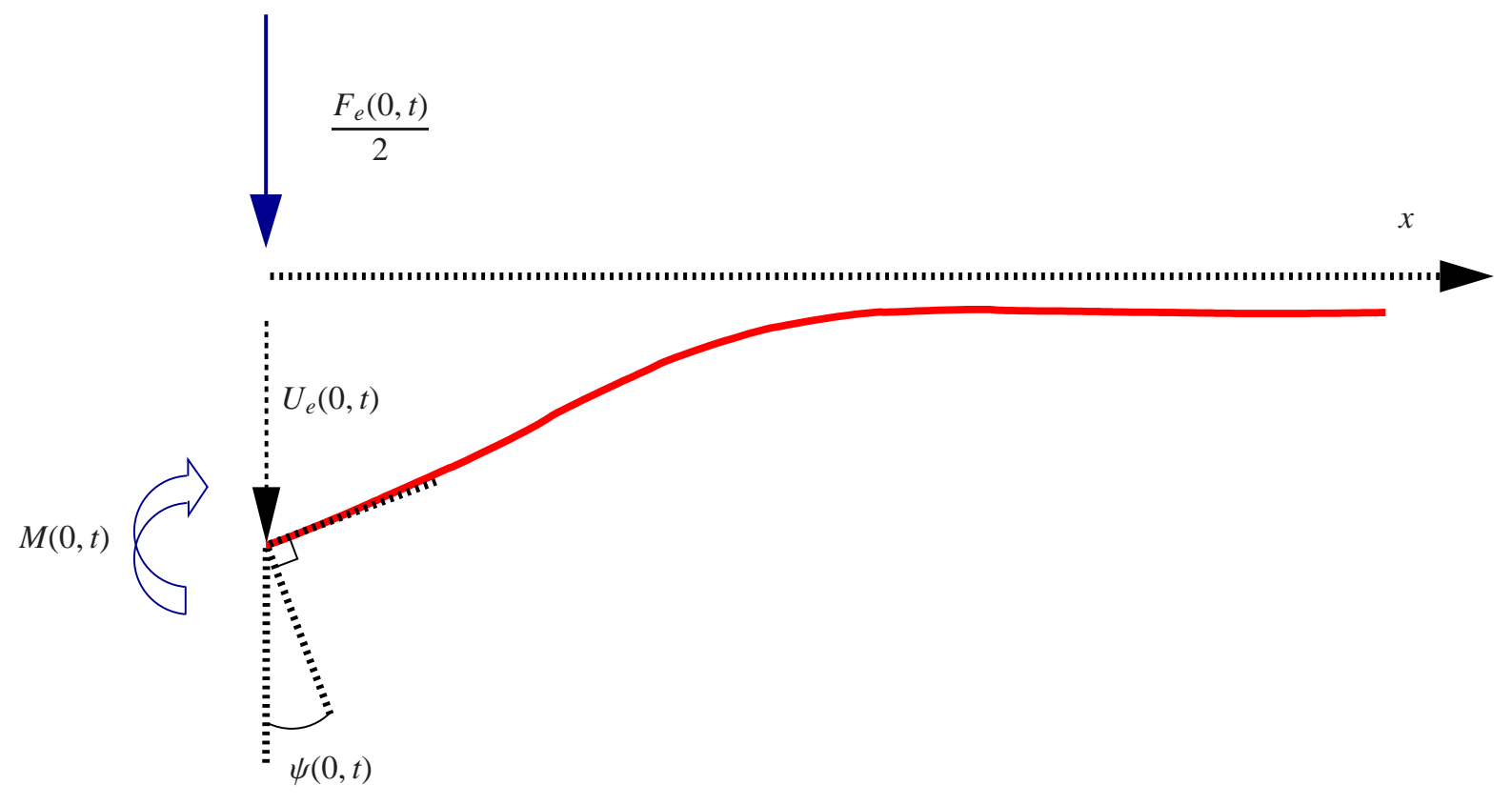

Figure 5: Beam and boundary conditions 


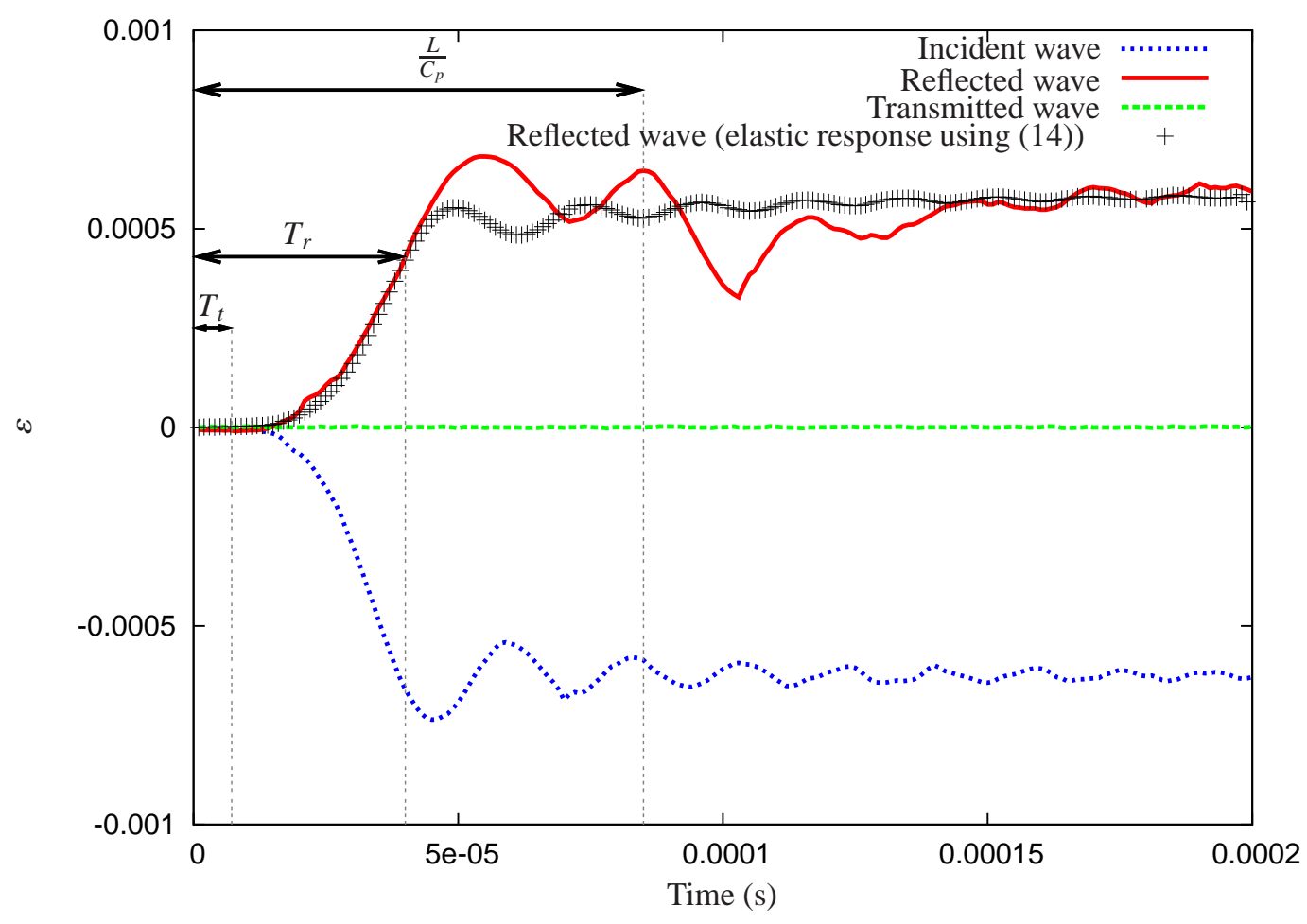

Figure 6: Waves time shifted on the basis of an elastic simulation of the reflected wave 

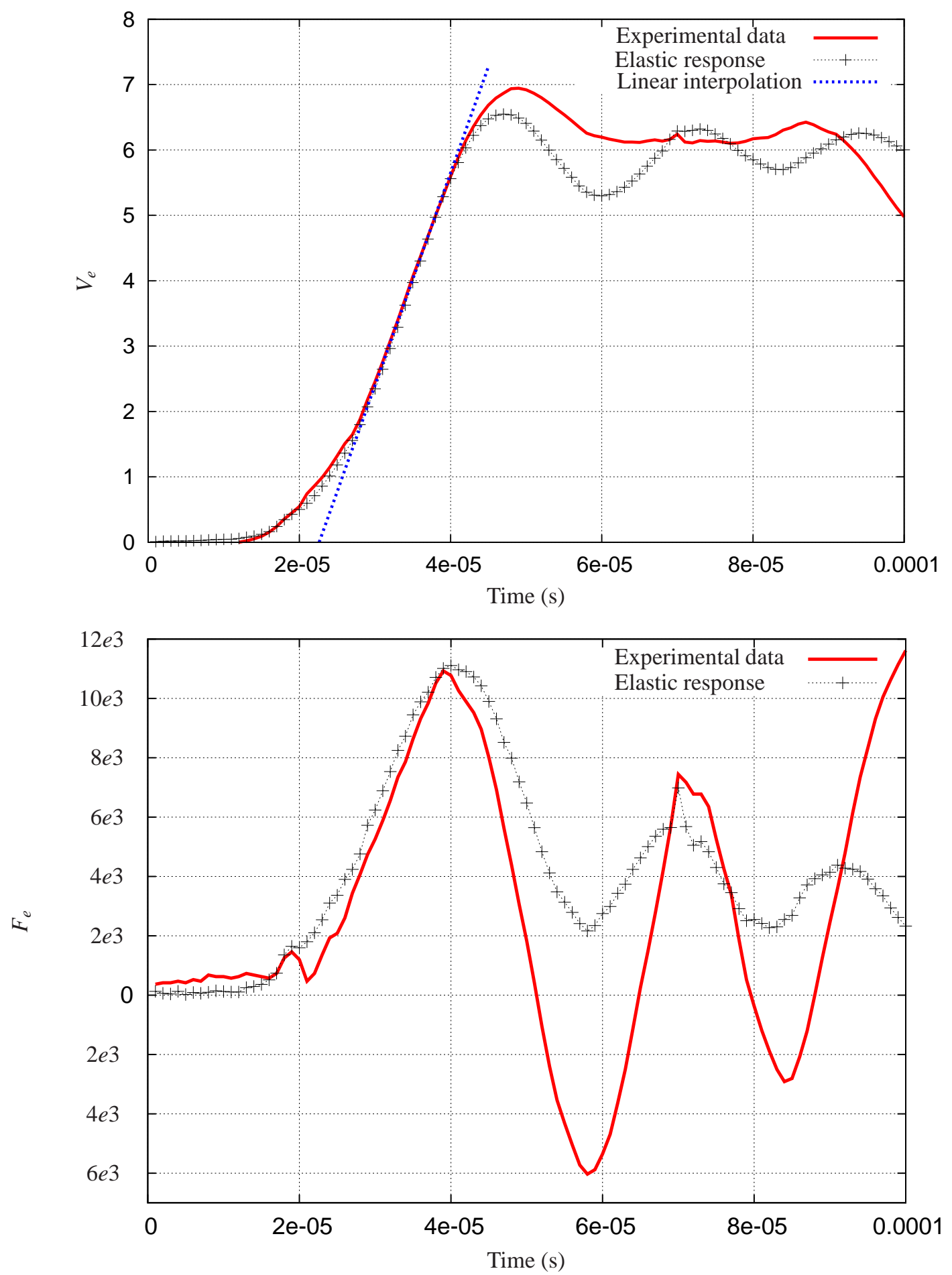

Figure 7: (a) Velocity at the impact point and linear interpolation used to determine the relevant strain rate reached before rupture, (b) Force at the impact point 


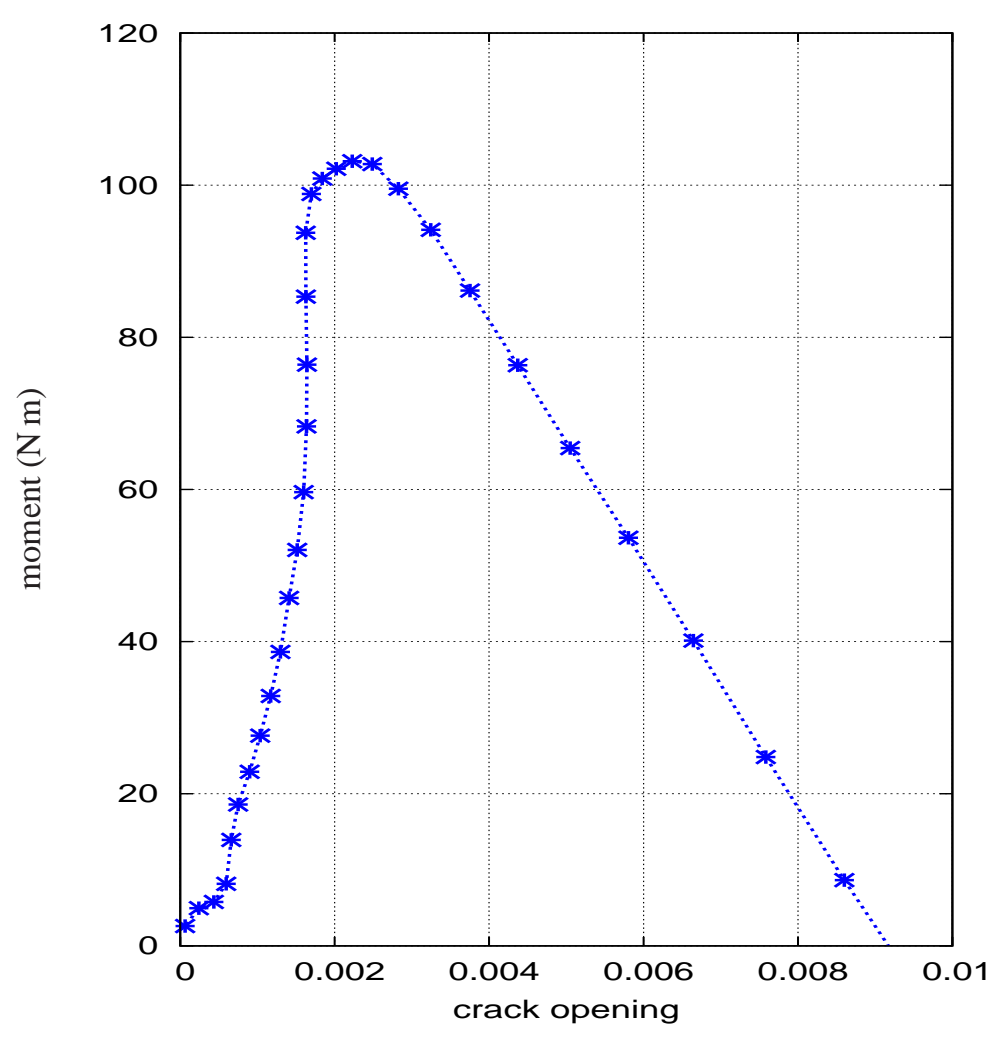

Figure 8: $M(0, t)=f(2 \psi(0, t))$ 


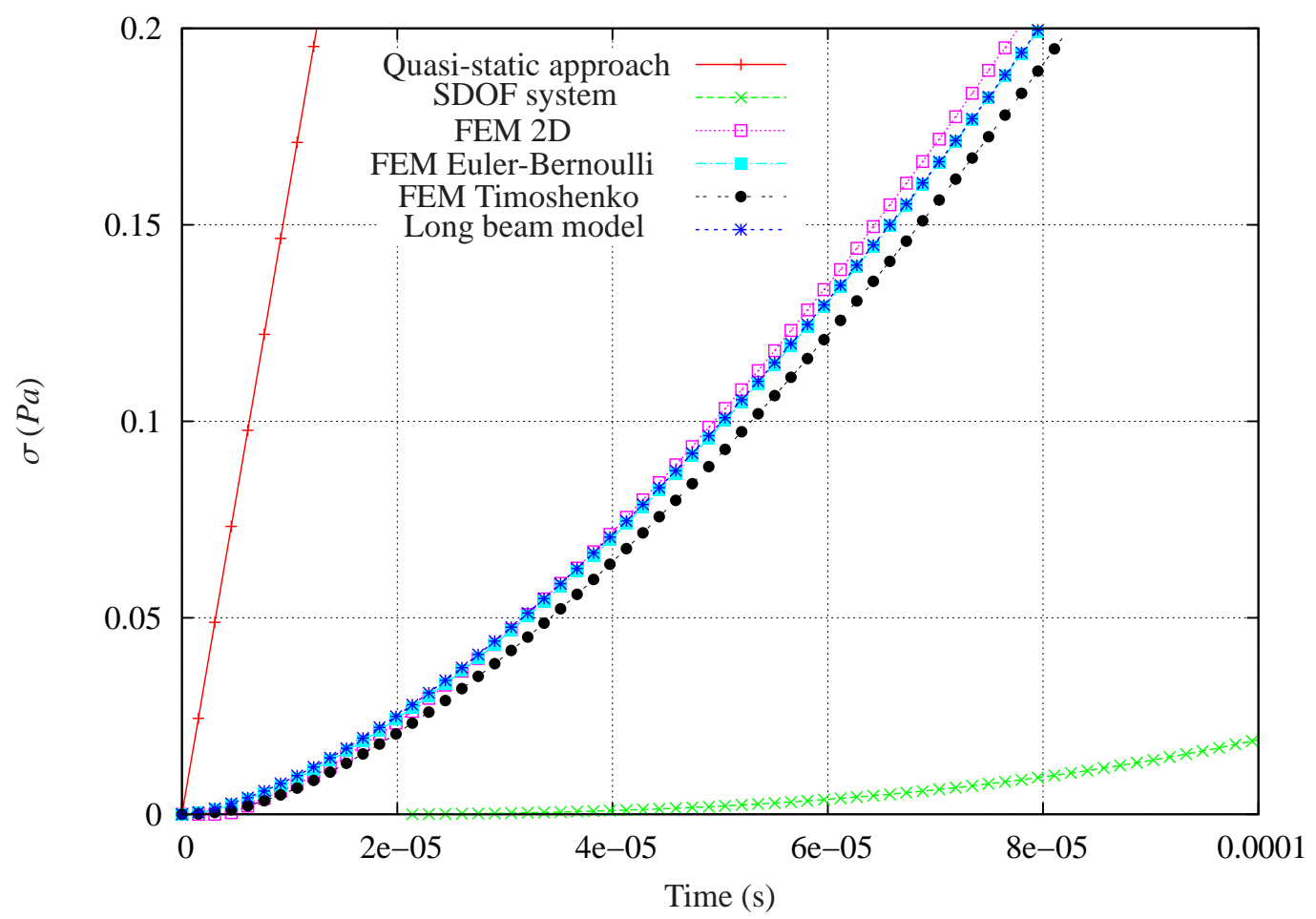

Figure 9: Maximum stress predicted by various models 


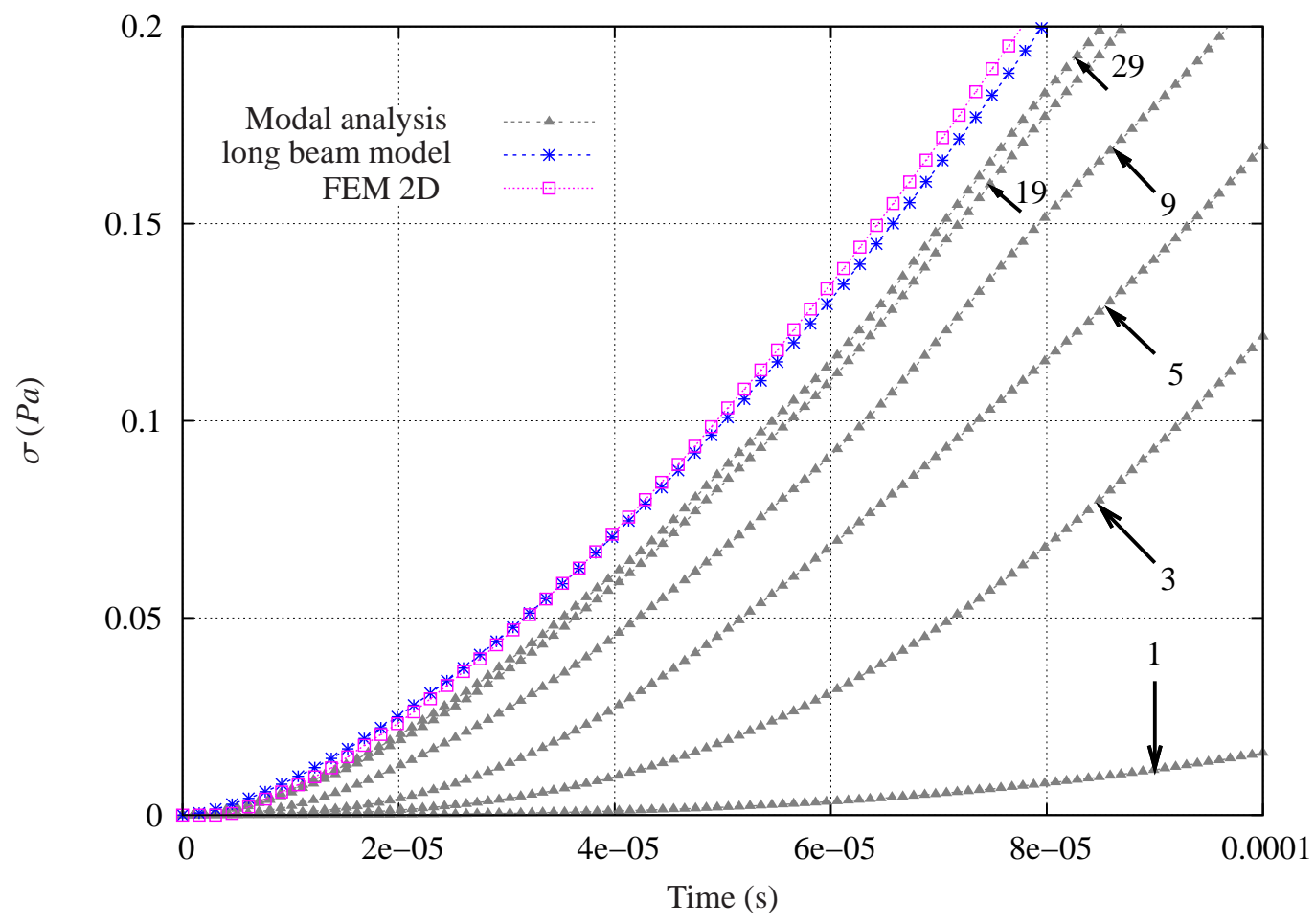

Figure 10: Convergence of the modal superposition analysis (black arrows precise the number of modes used) 